\title{
Visionen Realität werden lassen
}

\section{Das WikiLibrary Manifest und die WLB}

Ein offenes Wissensnetzwerk für alle - alles nur ein schöner Traum? Nein, es ist eine Vision, die es gilt, Realität werden zu lassen. Deshalb haben der gemeinnützige Verein Wikimedia Deutschland und die Deutsche Nationalbibliothek federführend das WikiLibrary Manifest unterzeichnet, eine Erklärung, in der Leitlinien und Maßnahmen für die Umsetzung dieses Zieles festgelegt werden. Als Kennwörter seien hier nur einige genannt: freie Lizenzen, Community Gardening, strukturierte Daten, gemeinsame Mindeststandards, Datenkompetenz, Wikibase, einfacher Datenaustausch, GLAM (Galleries, Libraries, Archives, Museums) (https://www.wikimedia.de/ projects/wikilibrary-manifest).

Wikimedia und Bibliotheken kooperieren schon lange, beispielsweise wenn Wikipedianer*innen bei der Texttranskription von digitalisierten Bibliotheksbeständen helfen oder Bibliothekar*innen im Projekt "\#1Lib1Ref" Referenzangaben für Wikipedia-Artikel liefern. Ein gemeinsames Ziel verbindet sie, nämlich freies Wissen zu fördern.

Auch die Führungskräfte der WLB haben sich klar für eine Unterzeichnung des WikiLibrary Manifests ausgesprochen. Die WLB ist motiviert, die Vision, ein „zuverlässiges, maschinenlesbares und gemeinschaftlich unterhaltenes Linked Open Data Netzwerk für Kunst, Kultur und Wissenschaft als tragfähige Grundlage für FAIRes Wissen“ zu unterstützen und umzusetzen.

Zu den Aufgaben der größten wissenschaftliche Bibliothek und Archivbibliothek für Baden-Württemberg zählen nicht nur die überregionale Informations- und Literaturversorgung, sie ist - außerhalb von Corona- und Umbauphasen - ein lebendiger Lernort und aktive Wissens- und Kultureinrichtung und beherbergt weltweit bedeutende Sammlungen. "Im Hintergrund" werden dafür umfangreiche Daten erfasst, angefangen von Titelbeschreibungen des Medienbestandes, über Sacherschließungsdaten und regionale Nachweise in der Landesbibliographie bis hin zu Digitalisaten und unikalen Datenbeständen der Sammlungen. Mit dieser Datenvielfalt verwaltet die WLB ein enormes Potential für eine vernetzte offene Wissenswelt, das vielfach noch ungenutzt "schlummert". Zum Beispiel, wenn es darum geht, Katalog-Daten als Open Data bzw. Linked Open Data für eine Weiternutzung zur Verfügung zu stellen. In anderen Arbeitsbereichen werden dagegen bereits „Mini“-Wissensnetzwerke praktiziert. Beispielsweise in Kooperation mit dem Landesarchiv BadenWürttemberg und dem landeskundlichen Informationssystem LEO-BW (https://www.leo-bw.de). In einem gemeinschaftlichen Datawarehouse werden hier verschiedenste Daten von Wissenschafts-, Kunst- und Kultureinrichtungen zusammengeführt und Informationen zu Personen, Orten und Regionen über Linked Data mittels Identifier aus der bibliothekarischen Gemeinsamen Normdatei (GND) miteinander verknüpft abrufbar. So sind die Daten der Landesbibliographie und ihrer Personendatei oder die der Bibliothek für Zeitgeschichte direkt mit anderen nichtbibliothekarischen Daten verlinkt. Gemeinsame Mindeststandards sind für die Umsetzung dabei ganz wichtig. Artefakte, Quellen und Literatur können so sammlungs- und einrichtungsübergreifend besser vermittelt werden.

Die GND aus der Bibliothekswelt ist aber nicht für alle Institutionen ein gleichermaßen geeignetes Werkzeug (vgl. hier auch Projekt GND für Kulturdaten (GND4C, https://www.dnb.de/DE/Professionell/ ProjekteKooperationen/Projekte/GND4C/gnd4c.html). Daher ist die Einrichtung einer Wikibase-basierten GND-Instanz sehr zu begrüßen, denn auch die WLB könnte diese nutzen, um nicht-GND-konforme Daten aus den Sammlungen oder Projekten abzulegen. AuBerdem kann die WLB offene Daten beispielsweise aus Wikidata für die maschinengestützte Verschlagwortung verwenden. Neben der Nachnutzung der Arbeit Anderer leistet die WLB in ihren Spezialgebieten (Region, Zeitgeschichte, Hölderlin, Stefan George) einen wichtigen Beitrag für den Aufbau eines umfassenden Daten-Ökosystems und Wissensnetzwerkes. Die Leitlinien des Manifests werden der WLB bei den aktuellen und künftigen Projekten als „Fahrplan" zur Umsetzung der Philosophie der FAIR-Data-Prinzipien (Auffindbarkeit, Zugänglichkeit, Interoperabilität und Wiederverwendbarkeit) dienen.

Anja Thalhofer 